\title{
Les fabriques du vivant
}

\section{Factories of the living}

\author{
Ruth Scheps ${ }^{1}$ \\ ${ }^{1}$ docteur en génétique moléculaire (The Weizmann Institute of Science, Rehovot, Israël) ; productrice à France \\ Culture et journaliste à la Radio Suisse Romande jusqu'en 2009 ; rédactrice en chef de la revue Mikhtav Hadash / La \\ Nouvelle Lettre jusqu'en 2019. rscheps@hotmail.com
}

RÉSUMÉ. Un livre (Xénobiologie, de Marie-Christine Maurel et Michel Cassé) et deux expositions (La Fabrique du vivant au Centre Pompidou, Formes vivantes au Musée national Adrien Dubouché à Limoges) ont témoigné en 2019 d'un intérêt convergent pour le vivant: la science l'étudie et le synthétise; l'art l'imagine et s'en inspire pour le représenter, le bio-art pour le recréer. La mythologie et la littérature ont révélé depuis longtemps la porosité des catégories de vivant et non-vivant dans l'imaginaire des peuples. Les expérimentations contemporaines menées par les scientifiques (biologistes, informaticiens, mathématiciens) et les créateurs (artistes, designers, architectes) tendent à estomper la séparation entre le vivant/naturel/sujet et le non-vivant/synthétique/objet. Elles donnent lieu à de nouvelles hybridations, des "vies partielles » dont les raisons d'être vont de la multifonctionnalité à visée écologique au questionnement philosophique sur le statut du vivant et la place de l'humain dans le monde numérique. Le présent article entend prolonger ce questionnement et attirer l'attention sur les implications éthiques des œuvres mixtes auxquelles la plupart de leurs créateurs n'accordent qu'une place restreinte.

ABSTRACT. In 2019, one book (Xénobiologie, by Marie-Christine Maurel and Michel Cassé) and two exhibitions ( $L a$ Fabrique du vivant at the Pompidou Center, Formes vivantes at the Musée national Adrien Dubouché in Limoges) displayed a convergent interest for the living : science studies and synthesizes it ; art imagines it and draws inspiration from it to represent it, bio-art to recreate it. Mythology and literature have revealed long ago the porosity between the categories of living and non-living in peoples' imagination. Contemporary experiments conducted by scientists (biologists, computer scientists, mathematicians) and creators (artists, designers, architects) tend to blur the split between the living/natural/subject and the non-living/synthetic/object. They give rise to new hybrids, "partial lives " whose raisons d'être extend from ecologically oriented multifunctionality to philosophical questioning on the living status and the place of the human within the numerical world. The present paper aims at extending this inquiry and drawing attention to the ethical implications of those hybrid works to which most of their creators only pay limited attention.

MOTS-CLÉS. Xénobiologie, vie partielle, biologie synthétique, bio-art, hybridation, cybernétique, auto-organisation, éthique.

KEYWORDS. Xenobiology, partial life, synthetic biology, bio-art, hybridization, cybernetics, self-organization, ethics.

\section{Introduction}

La France a accueilli en 2018-2019 trois événements liés au vivant et situés à la croisée des arts et des sciences : la publication du livre de Marie-Christine Maurel et Michel Cassé, Xénobiologie. Vers d'autres vies (Odile Jacob, 2018) ; une exposition au Centre Pompidou, La Fabrique du vivant (20 février - 15 avril 2019), troisième édition de la manifestation « Mutations/Créations », qui «propose de retracer une archéologie du vivant et de la vie artificielle dans la création artistique ${ }^{1}$; une exposition au Musée national Adrien Dubouché à Limoges, Formes vivantes ${ }^{2}$ (9 octobre 2019 - 10 février 2020). Un traitement conjoint de ces trois entreprises nous parait justifié dans la mesure où elles témoignent toutes d'intérêts convergents pour le vivant, non seulement tel qu'il se présente sur

\footnotetext{
${ }^{1}$ S. Lasvignes, B. Blistène et F. Madlener, préface du catalogue La Fabrique du vivant, éditions du Centre Pompidou, éditions HYX -2019 .
}

${ }^{2}$ Article en ligne de Jean-Charles Hameau et Kimberley Harthoorn, « Formes vivantes » (OpenScience, 16 mai 2019 ). 
Terre, dans ses formes réelles présentes ou passées, mais aussi tel qu'il pourrait exister ailleurs dans l'univers, tel que les mythes et la littérature l'ont décrit, enfin tel que les arts l'ont observé, imaginé et représenté tout au long de l'histoire.

Nous prendrons appui sur les approches spécifiques des organismes et systèmes vivants par les pratiques contemporaines de la biologie d'une part, des arts, du design et de l'architecture d'autre part. Nous tenterons ainsi de préciser la nature et les limites de ces approches théoriques et expérimentales, nous risquerons quelques considérations d'ordre sémantique au regard de ce qui est dit «vivant» et « humain », et nous ferons état de nos espoirs comme de nos craintes concernant leurs devenirs.

\section{Xénobiologie}

Le livre Xénobiologie, de la biologiste Marie-Christine Maurel et de l'astrophysicien Michel Cassé, est sous-tendu par une vaste ambition, aussi rigoureuse que poétique : embrasser l'ensemble des connaissances scientifiques actuelles sur les conditions de possibilité de la vie, dans toutes ses formes connues sur Terre, mais aussi ailleurs dans l'univers, comme le suggère le terme xénobiologie (littéralement, étude des formes de vie étrangères). Pour relever ce défi, les auteurs ont porté leurs regards sur trois terrains : le cosmos, la Terre, le laboratoire. De la composition physicochimique du cosmos, ils font ressortir les molécules organiques complexes qui pourraient avoir ensemencé notre planète. À partir des divers scénarios en vigueur, ils dressent un bilan des certitudes et des hypothèses sur les mécanismes évolutifs. Des formes actuelles du vivant, ils déduisent ce qui fait l'originalité de la vie sur Terre. Enfin, au cœur des laboratoires, ils passent au crible la biologie de synthèse, ses enjeux, ses acquis et ses dangers.

\section{De l'inerte au vivant}

Les nouvelles possibilités génétiques de transformation du vivant, voire de création de novo de cellules vivantes, ébranlent les distinctions traditionnelles entre le vivant (physico-biochimique) et l'inanimé (physico-chimique) et entre le naturel et l'artificiel.

Qu'il s'agisse de rendre compte des origines de la vie, des étapes de son évolution ou des expériences contemporaines visant à l'imiter, elle est de plus en plus souvent appréhendée en termes de continuum: l'évolution va de l'abiotique au pré-biotique, puis au proto-biotique, enfin au biotique (la vie pleinement vivante) ; la biologie de synthèse, elle, rend le naturel de plus en plus artificiel. Ce continuum, l'astrophysique nous invite à le penser encore bien au-delà de ses manifestations terrestres : jusque dans les étoiles - en particulier la nôtre, l'indispensable Soleil - et le «vide» cosmique dans lequel elles baignent. Effarés autant qu'émerveillés, nous découvrons à chaque page de nouvelles facettes de cet univers que le big bang semble avoir lancé dans une dilatation sans fin : feu, glaces, poussières et vide quantique frémissent d'énergie et de matière dont nous ne connaissons qu'une infime partie - comme pour les espèces vivantes, dont nous n'avons répertorié que 1 à $3 \%$ de celles présentes sur Terre de nos jours, lesquelles représentent environ $1 \%$ de toutes celles ayant existé depuis l'apparition de la vie, et dont une grande partie ont disparu avant d'avoir pu être découvertes.

L'arbre du vivant de ces dernières années ressemble bien peu à ses prédécesseurs des siècles passés, dont la structure évoquait en effet un arbre pourvu de racines, de troncs et de rameaux. C'est surtout du côté des origines que la classification a été remaniée : écartée, la sacro-sainte division binaire entre Procaryotes (unicellulaires sans noyau) et Eucaryotes (uni- ou pluricellulaires aux cellules généralement pourvues d'un noyau et souvent d'organites). La diversité du vivant s'envisage désormais comme une effloresence à partir d'un centre énigmatique, et sur un mode trinitaire : Bacteria, Archaea, Eucarya - la classification actuelle des Archées étant en pleine 
évolution. N'oublions pas cependant que cette diversité caractérise aussi le corps humain. Comme le rappelle Marie-Christine Maurel, Homo sapiens contient une multitude d'organismes associés à des virus et/ou des microbes : «L'être vivant ainsi défini, augmenté par mutualisme et non par greffes technologiques, n'est pas un cyborg ${ }^{3}$ organique mais une unité écologique qui s'origine dans les profondeurs de l'évolution et de son histoire ${ }^{4}$. »

L'évolution du vivant se caractérise par sa structure buissonnante et le foisonnement de ses formes - non seulement celles des organismes constitués mais bien en amont déjà, au niveau des molécules et même de leurs atomes. Plusieurs conditions physico-chimiques devaient être réunies pour que la vie puisse apparaître sur Terre, s'y maintenir et évoluer : une géochimie volcanique ; une atmosphère aux températures et aux pressions modérées, perméable aux rayonnements cosmiques ; une chimie diversifiée, notamment grâce aux chutes de météorites et à de fructueuses collisions avec des comètes. À travers ses récents développements (xénobiologie, exobiologie, biologie de synthèse), la biologie se tourne aujourd'hui vers ce passé inaccessible, mais aussi vers le futur imprévisible de la vie artificielle.

Concernant la vie connue sur Terre, plusieurs scénarios pré-biotiques ont été proposés : des impacts météoritiques auraient pu fournir les acides aminés nécessaires à la constitution des premières microcellules. Les conditions géochimiques favorables à leur émergence auraient pu être réunies en surface (petites mares tièdes, cristaux d'argile...) ou en profondeur (sources chaudes hydrothermales). Les simulations de ces processus en laboratoire confortent l'idée que la vie terrestre serait apparue non pas une seule fois mais à de nombreuses reprises. Cependant, les mécanismes par lesquels ces proto-organismes se sont dupliqués et reproduits - conditions nécessaires à l'évolution - ne sont pas encore élucidés. Les processus évolutifs eux-mêmes invitent à considérer le monde vivant comme inachevable, dans une suite ininterrompue d'extinctions et de renaissances.

\section{D'autres formes de vie}

À la recherche de formes de vie étrangères à celles connues sur Terre, donc différentes par leurs structures et leurs fonctions (tant somatiques que génétiques), l'exobiologie a d'ores et déjà inventé de nouvelles méthodes d'analyse pour les répertorier. Plus de 4100 exoplanètes ont été recensées à ce jour, qui pourraient remplir bon nombre des conditions nécessaires à l'apparition de la vie telle que nous la connaissons. Mais est-ce raisonnable de chercher à penser l'inconnu à partir du connu ? N'est-ce pas plutôt aux conditions nécessaires à l'apparition de la vie telle que nous ne la connaissons pas, que devraient s'intéresser les chercheurs? Pourront-ils relever ce défi quasi surhumain?

C'est du côté des nouvelles formes de vie - synthétiques ou semi-synthétiques - que les avancées sont les plus prometteuses... et les plus menaçantes.

En se fondant sur les recherches les plus récentes concernant les débuts de la vie sur Terre, la biologie de synthèse a créé de nouveaux génomes dont certains ont été insérés dans des organismes naturels. Les êtres vivants ainsi obtenus sont des sortes de chimères qui relèvent à la fois de la biologie ordinaire et de la xénobiologie. Présentées comme les promesses majeures du génie génétique, ces vies de synthèse sont destinées à améliorer le sort de l'humanité en contribuant à vaincre la faim dans le monde et les principales maladies qui affectent la santé humaine. Par quels

\footnotetext{
${ }^{3}$ Cyborg : organisme cybernétique, hybride de machine et de vivant, créature de la réalité sociale comme personnage de roman (définition de Donna Haraway, 1991).

${ }^{4}$ Marie-Christine Maurel, Xénobiologie, op. cit., p. 31.
} 
mécanismes ? Le génie génétique modifie l'ADN de certaines bactéries, les transformant ainsi en usines biologiques. Ces bactéries dites recombinantes, sont alors capables de produire des protéines humaines aux effets thérapeutiques (hormones, facteur de coagulation, etc.). En outre, la chirurgie génomique permet d'éliminer certains agents pathogènes, d'inventer de nouveaux traitements (DMLA, sida), de stimuler les rendements agronomiques ou la ponte des volailles et même de recréer des espèces perdues. Le CCNE (Comité Consultatif National d'Éthique) a cependant souligné les dangers de ces biotechnologies ${ }^{5}:$ « La connaissance et la compréhension croissantes du génome ouvrent la voie à des méthodes de plus en plus spécifiques et sophistiquées d'intervention sur l'ADN humain, ce qui n'est pas sans soulever de vastes questionnements éthiques tant dans le domaine des cellules somatiques (thérapie génique cellulaire) que de celui des cellules reproductrices ou de l'embryon (thérapie génique germinale). »

Les synthèses d'organismes vivants mi-artificiels, mi-naturels par imitation de processus naturels et hybridation d'éléments naturels et artificiels font en effet de leurs auteurs des apprentis-sorciers un risque brièvement évoqué par les auteurs - et il est à craindre que des chercheurs sans scrupules ne reconnaissent de limites à leur libido sciendi que celles imposées par les techniques du moment, dont la constante évolution ne cesse d'élargir le champ réel des expérimentations.

Claude Bernard le disait déjà en 1867 (deux ans après la découverte des lois de l'hérédité par Gregor Mendel, alors que le gène n'était encore qu'un concept abstrait) : «Je pense en un mot [...] que nous ferons apparaître des formes organisées qui existent virtuellement dans les lois organogéniques, mais que la nature n'avait pas encore réalisées. » La formulation de Claude Bernard, «des formes organisées qui existent virtuellement dans les lois organogéniques », justifie par avance les nouvelles formes vivantes susceptibles d'être fabriquées, en les admettant au sein de la Vie en général, sans distinguer à cet égard entre le naturel et l'artificiel. Claude Bernard se montre ici à la fois très moderne - il anticipe le processus contemporain d'effacement des frontières entre l'organique et le minéral, le naturel et le synthétique - et tout à fait de son temps - il ne peut imaginer qu'une nouvelle forme vivante soit autre chose que l'actualisation d'une virtualité préexistante. Autrement dit, il ne peut concevoir des vies radicalement « autres».

\section{Fabriques du vivant}

Les autres vies étudiées, synthétisées ou postulées par la xénobiologie, sont aussi l'objet de certaines formes d'art, qui font évoluer la notion de « vivant » en travaillant avec de nouveaux états intermédiaires d'artificialité. En témoignait récemment la fascinante exposition du centre Pompidou La Fabrique du vivant (20 février - 15 avril 2019) où l'on pouvait voir les œuvres récentes d'une cinquantaine de créateurs (artistes, designers, architectes), ainsi que des travaux de laboratoires scientifiques.

Dans son article liminaire du catalogue La Fabrique du vivant, Marie-Ange Brayer met d'emblée le doigt sur ce qui empêche désormais d'opposer les arts et les sciences, dès lors qu'ils concernent le vivant : "Aujourd'hui, à l'ère du numérique, la création se donne dans une interaction avec le domaine des sciences du vivant, la biologie synthétique, les sciences de l'informatique et les sciences cognitives ${ }^{6}$.» Nous nous proposons ici d'explorer les diverses modalités de cette interaction ainsi que leurs conséquences théoriques et pratiques dans tous les domaines concernés.

\footnotetext{
${ }^{5}$ Comité Consultatif National d'Éthique pour les sciences de la vie et de la santé, Avis 124 : «Réflexion éthique sur l'évolution des tests génétiques liée au séquençage de l'ADN humain à très haut débit. » Avis rendu public le 21 janvier 2016.

${ }^{6}$ Marie-Ange Brayer, « La fabrique du vivant » dans La fabrique du vivant. Mutations / Créations. Éditions du Centre Pompidou, éditions HYX - 2019. 
D’une manière générale, les visées contemporaines de l'art et des sciences du vivant convergent, voire s'entrecroisent, la biologie de synthèse créant de la « vie artificielle » et le bio-art s'inspirant des processus dynamiques du vivant. Ces convergences se manifestent à plusieurs niveaux et de différentes façons.

\section{Multiplication des possibles}

En xénobiologie comme dans les arts qui imitent, représentent ou transforment le vivant, il s'agit autant de multiplier les possibles que d'en contrôler l'expression. Les biologistes multiplient les possibles en simulant les conditions physico-chimiques ayant conduit à l'émergence de la vie sur Terre, en créant de nouveaux acides nucléiques voire de nouvelles formes de vie biosynthétiques, et en recréant des espèces vivantes disparues ; les artistes, artisans et designers, en façonnant des objets qui représentent ou font écho à certaines formes vivantes ${ }^{7}$, en créant des systèmes partiellement vivants voire de nouveaux systèmes cognitifs mixtes. Concernant les formes inspirées par le vivant, l'art contemporain de la céramique témoigne d'un riche imaginaire organique ${ }^{8}$ et de savoirs empiriques sur les interactions entre forme et matière, qui rejoignent les études scientifiques sur la morphogenèse. Quant aux productions d'entités semi-vivantes, elles peuvent être contrôlées en amont (par programmation, numérisation, impression 3D) ou en aval (par intervention sur les caractéristiques physiques de l'environnement). Quoi qu'il en soit, l'extension du domaine du vivant dépend toujours de la mise en œuvre de nouvelles biotechnologies, parfois utilisées de manière conjointe.

Dans la fabrique génétique du vivant, l'adage « un gène, une protéine » a longtemps prévalu, mais c'est la découverte qu'un même gène peut coder des protéines différentes selon le type de cellules où il s'exprime (années 1970), qui a permis de rendre compte de l'immense diversité des molécules. Dans la fabrique artistique du vivant, de nombreux objets de design et projets architecturaux sont eux aussi multifonctionnels, dans une perspective souvent écologique : c'est le cas des architectes français Anouk Legendre et Nicolas Desmazières ${ }^{9}$, dont les constructions, loin d'être passives, produisent de l'énergie (par la photosynthèse ou le photovoltaïque) et récupèrent les déchets ou les eaux usées en vue d'autres usages. C'est également vrai pour la designer Neri Oxman ${ }^{10}$, qui fabrique des objets et des bâtiments à la fois durables et biodégradables, à partir de biomatériaux constitués de micro-organismes transformés de manière synthétique et qui interagissent avec les paramètres physiques de leur environnement - comme son œuvre Silk Pavilion, «construction vivante » élaborée par des milliers de vers à soie. D'autres artistes se situent sur un terrain plus philosophique, et questionnent la définition même du vivant ou le statut de l'humain dans un monde envahi par le numérique.

\footnotetext{
${ }^{7}$ Jean-Charles Hameau et Kimberley Harthoorn, op. cit.

${ }^{8}$ Jean-Charles Hameau et Kimberley Harthoorn, op. cit, sur les concrétions florales de Johan Creten, les grotesques agglutinés de Michel Gouéry, les bestiaires émaillés de Jean Girel et les micro-organismes d'Arnold Annen.

${ }^{9}$ Nicolas Desmazières et Anouk Legendre ont fondé en 2000 l'agence d'architecture et de design XTU Architects. Avec leur équipe ils conçoivent des projets architecturaux en lien avec les sciences et la biologie dans une perspective écologique.

${ }^{10}$ Neri Oxman travaille à la croisée du design computationnel, de la fabrication numérique, de la science des matériaux et de la biologie synthétique. Au sein du MIT Media Lab à Boston, elle a créé le laboratoire de recherche The Mediated Matter Group. Elle a inventé le concept de Material Ecology, qui fait de la fabrication, du calcul numérique et du matériau lui-même, appréhendé comme « logiciel », des éléments inséparables du design. Son œuvre Silk Pavilion (2013) a été réalisée au MIT.
} 


\section{Continuités et hybridations}

L'interpénétration des domaines du vivant/sujet et de l'artificiel/objet concerne des champs très variés : littérature et sciences humaines, arts plastiques, informatique, sciences de la matière et de la vie. Elle les a transformés de manière spécifique et à des périodes historiques différentes.

Dans l'imaginaire traditionnel de la plupart des peuples autochtones, la matière inorganique n'a jamais été opposée à la matière vivante, chacune étant susceptible de devenir l'autre. Dans le monde latin antique, Les métamorphoses d'Ovide témoignent abondamment de cette plasticité. Plus récemment en Occident, le golem (en hébreu, «informe » ou «inachevé ») est, dans la mystique et la mythologie juives, un être artificiel généralement humanoïde, fait d'argile, muet et dépourvu de libre-arbitre, façonné afin de servir son créateur. Dans une version populaire de sa légende, il naît de la terre glaise après que quatre sages, figurant les quatre éléments, lui ont transmis leurs attributs ; sur son front figure le mot emet (« vérité ») qui devient, lorsque sa première lettre est effacée, met («mort ») : l'homme artificiel est retourné à la poussière. Selon quelques auteurs, la figure du golem aurait pu inspirer certaines créatures de l'imaginaire moderne (monstre de Frankenstein, Superman). Dans le monde scientifique du XX ${ }^{\mathrm{e} m e}$ siècle, le golem a également été source d'inspiration, par exemple pour Norbert Wiener, fondateur de la cybernétique (1940-1960) ${ }^{11}$. Avec la notion de rétroaction (feedback), Wiener a tenté d'unifier les domaines émergents de l'automatique, l'électronique et la théorie mathématique de l'information; dans son article «Pure Patterns in a Natural World $»^{12}$, il a cherché à unifier l'art et la science; enfin, dans son dernier ouvrage intitulé God \& Golem $^{13}$, il a décrit conjointement «la machine dans son rapport à l'humain et l'humain lui-même comme machine possible, comme devenir-Cyborg ${ }^{14}$. Wiener était intellectuellement tenté par ces figures qui révélaient le statut paradoxal de l'être humain, plongé «entre nature et technique, entre Dieu et Bête ${ }^{15}$, mais il était tout autant effrayé par la monstruosité de ce post-humain, qu'il avait pourtant contribué à penser.

L'art contemporain va redécouvrir et mettre à l'honneur l'idée ancienne de continuité entre le vivant et le non-vivant, pour le coup beaucoup moins inerte que ce qu'en disait la science des siècles passés. Le céramiste Daniel de Montmollin (né en 1921) envisage ainsi l'art de la poterie comme « la confluence entre le minéral et le vivant, révélant, à l'échelle atomique, les processus d'une "biologie transfigurée" ${ }^{16}$. Les céramistes, frappés par les ressemblances et la porosité qu'ils observent entre matières minérales et organismes vivants, vont jusqu'à mettre en doute les catégories autrefois séparées de vivant et de non-vivant, témoignant ainsi d'un «nouvel imaginaire du vivant ». Et depuis la fin des années 1960, les productions du bio-art témoignent elles aussi de cette continuité.

\footnotetext{
${ }^{11}$ Norbert Wiener (1894-1964), La cybernétique : Information et régulation dans le vivant et la machine (trad. de l'anglais par Ronan Le Roux, Robert Vallée et Nicole Vallée-Levi), Seuil, 2014, « Introduction ».

${ }^{12}$ Norbert Wiener, "Pure Patterns in a Natural World », catalogue de l'exposition The New Landscape in Art and Science, éd. G. Kepes, Boston, 1956.

${ }^{13}$ Norbert Wiener, God \& Golem Inc. Sur quelques points de collision entre cybernétique et religion, éd. de l’Éclat, 2001 (rééd. 2016 : Préface de Charles Mopsik. En postface : « Le Golem de Prague et le Golem de Rehovot » (1965) par Gershom Scholem. Traduit de l'anglais par Christophe Romana \& Patricia Farazzi).

${ }^{14}$ Sara Touiza-Ambroggiani, Golem et Cyborg dans l'imaginaire cybernétique de Norbert Wiener. Conférence organisée par Figura, le Centre de recherche sur le texte et l'imaginaire. Montréal, Université du Québec à Montréal, 31 octobre 2017.

${ }^{15}$ Sara Touiza-Ambroggiani, ibid.

${ }^{16}$ Jean-Charles Hameau et Kimberley Harthoorn, "Formes vivantes ", op. cit. 
En 1968, le sculpteur, théoricien et critique Jack Burnham (1931-2019) définit le système comme un «complexe de formes visibles et invisibles dans une relation stable » ${ }^{17}$, brouillant ainsi la distinction entre sujet et objet, et entre système vivant et pratique artistique ; il déclare même que la sculpture sera bientôt remplacée par des systèmes simulant le vivant. En 1971, dans son œuvre Microorganism Enclosure, Alan Sonfist ${ }^{18}$ capture des bactéries et des champignons dans un environnement fermé, désormais programmé par l'évolution des phénomènes naturels de croissance et décomposition, et au sein duquel sujet et objet se confondent.

Dans cette «fabrique du vivant», les substrats vivants des œuvres sont surtout des microorganismes, du mycélium de champignons ou des plantes; très rarement des animaux vivants - à l'exception notable du lapin fluorescent GFP Bunny (2000) d'Eduardo Kac, promoteur de l'art transgénique $^{19}$. L'humain lui-même n'y est représenté que par quelques lignées cellulaires..., jusqu'au début des années 1990, lorsque le sociologue du design Ezio Mazzini propose de remplacer la frontière séparant le sujet de l'objet par des «membranes osmotiques ${ }^{20}$, ouvrant ainsi la voie au «neurodesign» dans lequel l'objet (désormais intrinsèquement lié au sujet) émanerait d'un «continuum d'interactions entre le cerveau humain et ses artefacts technologiques, basé sur le biofeedback neuronal et la digitalisation de la cognition humaine ${ }^{21}$. » D'autres chercheurs poursuivent ce type d'expérimentations et en 2008, une équipe britannique de l'université de Reading développe «Gordon », un robot hybride animal/machine contrôlé par un cerveau composé de neurones de rats cultivés.

$\mathrm{Au}$ début du $\mathrm{XXI}^{\mathrm{ème}}$ siècle, les expérimentations artistiques d'hybridations se multiplient : en 2000, les artistes Oron Catts et Ionat Zurr élaborent des entités «partiellement vivantes » ${ }^{22}$, qui estompent la différence entre sujet et objet, ou entre solide et liquide. L'originalité de ces œuvres est que leur «vie » nécessite une forme d'assistance/maintenance qui s'apparente à des soins (« rituels d'alimentation ou de mise à mort »). En 2018, le designer Hongjie Yang, aspirant à transcender la frontière entre vivant et artificiel à travers des objets hybrides, crée un Semi-Human Vase constitué de cellules humaines implantées sur une structure imprimée en 3D. "Ces créations représentent, selon lui, une " "nouvelle vision du sublime" qui suscite autant l'admiration que l'effroi devant la connexion étrange entre le soi et l'objet ${ }^{23}$.» Un cap supplémentaire a été franchi : il ne s'agit plus de micro-organismes ou de fragments de plantes, mais de cellules d'origine humaine. Celles-ci sont certes utilisées depuis longtemps en médecine biologique (cultures de tissus, fécondation in vitro, etc.). Mais la notion de «sublime semi-humain » pose pour le moins question, quelle que soit sa

\footnotetext{
17 Jack Burnham, Beyond Modern Sculpture. The Effects of Science and Technology on the Sculpture of this Century, George Braziller, New York, 1968, p. 372.

${ }^{18}$ Alan Sonfist, Microorganism Enclosure, 1971. Photographie de I'installation dans J. Benthall, Science and Technology in Art Today, Londres, Thames \& Hudson, 1972, p. 16.

${ }^{19}$ La fabrique du vivant, op. cit., « GFP Bunny », p. 175.

${ }^{20}$ Ezio Manzini, Artefacts. Vers une nouvelle écologie de l'environnement artificiel. Paris, Centre Georges Pompidou, Les Essais, 1991, p. 30.

${ }^{21}$ Voir Pierre Cutellic, Fabien Lotte, eCAADe Computation and Performance, TU Delft, ed. R. Stouffs, S. Sariyildiz, 2013.

22 Oron Catts, lonat Zurr \& Guy Ben-Ary, "Que/qui sont les êtres semi-vivants créés par Tissue Culture \& Art ", dans L'art biotech', Filigranes éd., 2003. Les Tissue Culture \& Art(ificial) Wombs, Semi-Living Worry Dolls (2000) sont des entités « semivivantes ", inspirées de poupées amérindiennes, premières sculptures d'ingénierie tissulaire présentées vivantes dans une institution.
}

${ }^{23}$ La fabrique du vivant, op. cit., " Hongjie Yang », p. 234. 
justification : philosophique (effacer la frontière entre le vivant humain et l'artificiel) ou esthétique (atteindre une beauté d'ordre «transcendant»). Dans la même veine, la bio-artiste Špela Petrič interroge la relation entre système vivant et non-vivant. Pour réaliser son œuvre Ectogenesis : PlantHuman Monsters $(2016)^{24}$, elle a mélangé des embryons végétaux avec des hormones humaines, modifiant ainsi la structure épigénétique de la plante, qui a développé une morphologie étrange voire monstrueuse.

Face à ces œuvres dérangeantes qui visent - et parfois atteignent - tantôt au sublime, tantôt au monstrueux, chacun est renvoyé à ses propres perplexités touchant aux limites du vivant et à la finalité de l'art.

\section{Quel statut pour les « vies partielles »?}

\section{Des œuvres autonomes}

En science comme en art, travailler avec le vivant a toujours impliqué d'être confronté à sa part imprévisible - que l'approche statistique peut réduire mais ne supprime pas. Dans leur désir de connaître le monde (vivant et non-vivant) jusqu'à s'en rendre «maitres et possesseurs », les scientifiques l'ont profondément transformé, et continuent de le faire à un rythme de plus en plus rapide. Vers la fin du XIX ${ }^{\text {ème }}$ siècle, l'utilisation de la génétique à des fins esthétiques (plantes hybrides ornementales) a étendu cette emprise sur la nature à des formes sublimées du vivant. Désormais, au regard des arts plastiques et dans leur matérialité, les formes vivantes ne sont plus seulement des sources d'inspiration pour les peintres et les sculpteurs, mais atteignent elles-mêmes au statut d'œuvres.

En contrepoint à l'idéal de la maîtrise et du contrôle qui avait gouverné les arts et les sciences jusqu'au début du $X{ }^{\text {ème }}$ siècle, les tenants du modernisme se sont davantage intéressés à la dimension auto-organisatrice du vivant - également célébrée par Gaston Bachelard dans La Terre et les rêveries de la volonté (1948) : "Tout m'est pâte, je suis pâte à moi-même, mon devenir est ma propre matière, ma propre matière est action et passion, je suis vraiment une pâte première. » Dans les années 1930, les chercheurs, artistes et architectes des avant-gardes étudient l'auto-génération des formes cristallines et dans les années 1960, quelques artistes, proches de l'art cinétique, se saisissent d'outils technologiques pour créer des systèmes autonomes. L'artiste Gustav Metzger met ainsi en œuvre un «art auto-créatif » (pendant de son « art auto-destructif » de 1959), dans lequel «il adopte comme principe, l'autonomie du processus à l'égard de celui qui l'initie et sa transformation imprévisible dans la durée en "permanente (auto)genèse" ${ }^{25}$ »-par exemple, en expérimentant sur les effets colorés de cristaux liquides modifiés par la température. En 1972, dans le sillage des notions de «système ouvert» (Ludwig von Bertalanffy) puis de «rétroaction» cybernétique (Norbert Wiener), au cours d'un séminaire de recherche de l'Université de Santiago, le neurobiologiste et spécialiste des sciences cognitives Francisco J. Varela présente avec Humberto Maturana le concept d'《autopoïèse $»^{26}$, que Varela précisera dans son livre Autonomie et

\footnotetext{
${ }^{24}$ Ectogenèse : gestation dans un utérus artificiel.

${ }^{25}$ Thierry Raspail, " Gustav Metzger, l'auto-création... », dans Mathieu Copeland (dir.), Gustav Metzger : auto-creative art, p.4. Catalogue d'exposition (Lyon, Musée d'art contemporain, 15 février - 14 avril 2013), Lyon, Musée d'art contemporain de Lyon ; Lagny-sur-Marne, Mathieu Copeland éditions ; Dijon, Les Presses du Réel, 2013.
}

${ }^{26}$ Autopoï̀se (définition simplifiée) : propriété d'un système de se produire lui-même, en permanence et en interaction avec son environnement, et ainsi de maintenir son organisation (structure) malgré son changement de composants (matériaux). 
connaissance $^{27}$. Dans la même mouvance, au MIT's Architecture Machine Group, dirigé par Nicholas Negroponte (1967-1985), certaines œuvres s'auto-engendrent au sein d'un environnement avec lequel elles interagissent - comme la machine $S e e k^{28}$, qui reconstruit inlassablement un terrain de jeu aménagé pour de petits rongeurs. En 2016, Philippe Parreno active une installation monumentale à la Tate Modern, Anywhen, contrôlée de manière aléatoire par un logiciel réagissant aux influx électriques de levures contenues dans un bioréacteur caché sur place. Ici, c'est l'exposition tout entière qui s'auto-organise. "L'exposition conçue par Parreno est un organisme hybride qui existe, quelque part, au croisement de la mécanique et de la biologie, de l'électronique et de la nature, de la musique et de l'image, entre l'artiste et son public ${ }^{29}$."

En faisant de l'autonomie et de l'auto-engendrement, des caractéristiques souhaitables de leurs œuvres systémiques, certains artistes se sont partiellement dessaisis du contrôle qu'ils exerçaient sur leurs productions. Ils assument ce renoncement au nom d'une nouvelle esthétique évolutive, inspirée par le vivant, et qu'ils valorisent davantage que les esthétiques figées du passé.

\section{Nommer ce qui existe}

On observe depuis quelques décennies une réticence croissante chez les biologistes comme chez les bio-artistes, à utiliser la notion de vie. Des spécialistes des origines de la vie déclarent même qu'ils n'en savent pas grand-chose ${ }^{30}$ et se réfèrent plus volontiers aux formes de vie (ibid.), une dénomination assurément moins essentialiste. Dans le catalogue de l'exposition La fabrique du vivant, les artistes cités ont tous remplacé vie par vivant, comme si la notion de vie charriait encore un transcendantalisme révolu. Mais voici que vivant est menacé à son tour : "La robotique collaborative, l'intelligence artificielle et la simulation numérique sont sur le point d'engendrer de nouveaux modèles cognitifs qui rendront obsolète la notion de vivant ${ }^{31}$. », sauf à le réduire à sa matérialité machinique ou synthétique: "Le vivant synthétique donne forme et s'hybride avec la

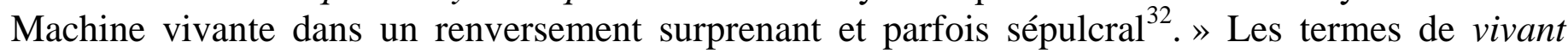
synthétique et de machine vivante ne sont plus des oxymores... Reste le terme d'organisme, lui aussi matérialiste à souhait. L'artiste, designer et critique Alexandra Daisy Ginsberg propose d'ailleurs de «concevoir des organismes de la même manière que nous dessinons des produits ${ }^{33}$.» Concevoir des organismes, serait-ce plus éthiquement correct que créer des êtres vivants? Il semble bien loin, l'être vivant jadis étudié par les naturalistes et célébré par les poètes. Aujourd'hui, amputé

\footnotetext{
${ }^{27}$ Francisco J. Varela, Humberto Maturana, et R. Uribe, “Autopoiesis: The Organization of Living Systems, its Characterization and a Model", Biosystems, vol. 5, 1974, p. 187. Cf. H. Maturana et F. Varela, Autopoiesis and Cognition: The Realization of the Living (Appendice: "The Nervous System"), Boston, D. Reidel, 1980. F. J. Varela, Autonomie et connaissance, Essai sur le Vivant, Paris, Seuil, 1989 (1980).

${ }^{28}$ Seek de Nicholas Negroponte : installation présentée pour la première fois en 1970 à New York, au Jewish Museum. Vidéo visible sur https://d-503.com/seek/.

${ }^{29}$ " Anywhen, ou la métamorphose du Turbine Hall, Tate Modern, par Philippe Parreno », Connaissance des arts, 15.02.2017.

${ }^{30}$ Cf. Marie-Christine Maurel et Michel Cassé : « Mais sait-on au moins ce que signifie la vie ? Tout au plus on la vit. Nous n'en savons pas plus de la vie que l'oiseau du vol, mais nous volons. ", dans Xénobiologie. Vers d'autres vies, op. cit., p. 18.

${ }^{31}$ Marie-Ange Brayer, « La fabrique du vivant », op. cit., p. 75.

32 Olivier Zeitoun, « Sculpter le vivant », catalogue La fabrique du vivant, op. cit., p. 88.

33 Alexandra Daisy Ginsberg, "Design as the Machines Come to Life », dans Alexandra Daisy Ginsberg, Jane Calvert, Pablo Schyfter, Alistair Elfick, Drew Endy, Synthetic Aesthetics. Investigating Synthetic Biology's Design on Nature, Cambridge, Mass., Londres, The MIT Press, 2014.
} 
de son être, c'est un vivant chosifié. Quant à la notion de sujet (humain), elle tend à disparaître en tant que telle, au profit des divers sujets-objets élaborés par les artistes comme par les scientifiques. Un exemple spectaculaire de ce type de mélanges est fourni par l'artiste Pamela Rosenkranz avec son monochrome « vivant » Skin Pool ${ }^{34}$, qui présente le sujet humain comme « une trace fluide », un composé organique indissociable des matériaux synthétiques avec lesquels il se confond.

\section{Questions éthiques}

Si nous nous adressons de manière plus frontale aux aspects éthiques des recherches et des créations sur le vivant, le paysage est contrasté. Dans les sciences du vivant, les avancées médicales et agronomiques des biotechnologies, qui promettent d'améliorer globalement la condition humaine, vont de pair avec des incertitudes sur lesquelles le CCNE a attiré l'attention (cf. supra). Au sein des arts $d u$ vivant, le cas particulier de la céramique, située à la croisée de l'art, de l'ingénierie et de la biologie, milite en faveur d'une continuité entre l'inorganique et le vivant, qui nous permet d' « entrevoir des rapports d'empathie avec les autres membres de la communauté du vivant ${ }^{35}$. » Cependant, dans le catalogue La fabrique du vivant, les textes présentant les bio-artistes soucieux d'éthique, expédient ce versant de leurs travaux en quelques formules standardisées : les œuvres des artistes, designers et architectes en question, sont censées «interroger » la définition du vivant, la nature du vivant modifié, la place de l'humain ou encore, ce que signifie être humain. Seule l'artiste Julia Lohmann ${ }^{36}$, qui conçoit son travail comme un «baromètre éthique », a droit à une présentation plus fournie en la matière : «Elle enquête et cherche à critiquer les systèmes de valeurs éthiques à la base de la relation entre l'homme et la nature. [...] Lors d'une résidence au Victoria \& Albert Museum de Londres, elle crée le Department of Seaweed, une plate-forme transdisciplinaire tournée vers l'exploration des algues marines en tant que ressource durable. » Dans son article déjà cité, Marie-Ange Brayer se demande (p. 72) : «Si les outils numériques de simulation générative autorisent la recréation du vivant, la question qui se pose désormais, est : Comment programmer le vivant ? ^Autoriser la recréation du vivant: le terme est employé ici au sens restreint de permettre techniquement. Mais qu'en est-il du bien-fondé éthique de cette «recréation » et de l'idée, déjà partiellement mise en œuvre, de «programmer le vivant»? Cette question rejoint celle posée par l'historien d'art et curateur Emanuele Quinz ${ }^{37}$ sur le statut du «vivant» dans les démarches artistiques qui s'inspirent des modèles cybernétiques et font cohabiter dans un même « écosystème » éléments organiques et inorganiques.

En 1991, Ezio Manzini déclarait : «Essayons d'imaginer [...] des objets qui, comme un arbre, soient appréciés pour ce qu'ils sont autant que pour ce qu'ils font, des objets qui rendent un service et réclament des soins ${ }^{38}$. » Ce que demande ici Manzini, c'est que nous, humains conscients de notre

\footnotetext{
${ }^{34}$ Skin Pool (2019, collection du Mnam-Cci, Centre Pompidou) : bassin circulaire en acier et PVC composé d'eau, de cellulose, de peinture acrylique et de colorant alimentaire. Le liquide monochrome, qui interagit avec les microbes présents dans l'air ambiant et ceux agités par les spectateurs, change sans cesse de couleur et d'aspect, et renvoie l'humain à sa nature organique et métabolique.

${ }^{35}$ Jean-Charles Hameau et Kimberley Harthoorn, op. cit.

${ }^{36}$ Julia Lohmann : travaille avec des matériaux inhabituels, d'origine souvent animale ou végétale (algues, estomac, papier, etc.). La fabrique du vivant, op. cit., p. 184.

${ }^{37}$ Emanuele Quinz, "Préface. Conceptual focus » dans Jack Burnham et Hans Haacke, Esthétique des systèmes, Les Presses du Réel, 2015, p. 25.

${ }^{38}$ Ezio Manzini, Artefacts. Vers une nouvelle écologie de l'environnement artificiel, Paris, Centre Georges Pompidou, Les Essais, 1991, pp. 181-182.
} 
humanité, devenions capables d'aimer les objets auxquels nous prêtons une ontologie. Cette demande est-elle si exorbitante? N'avons-nous pas assisté il y a quelques décennies à un engouement planétaire pour les Tamagotchis, ces animaux de compagnie virtuels ? Et ne considérons-nous pas nos ordinateurs portables comme des partenaires de vie dont nous chérissons les performances («ce qu'ils font ») et l'apparence («ce qu'ils sont») ? N'aimons-nous pas leur esthétique et leur légèreté, et ne blâmons-nous pas leurs défauts (lenteur, bugs), considérés quasiment comme des maladies?

Tout comme la xénobiologie, les arts du vivant proposent, bricolent et accueillent d'autres vies. Or ces vies nouvellement découvertes ou fabriquées, ne sont pas indépendantes de nos propres vies - de la structure de nos organismes jusqu'aux vies que nous menons en tant que telles, et en interaction avec les autres existants qui peuplent la Terre. Ce dernier point a conduit plusieurs penseurs contemporains à revaloriser le statut de non-humain.

Bruno Latour prend les non-humains très au sérieux. Dans son Enquête sur les modes $d^{\prime}$ existence $^{39}$, il considère qu'avec le développement des sciences et des techniques, les attachements entre humains et non-humains étant devenus de plus en plus intimes, il s'agit de penser vraiment ces nouveaux rapports et d'en faire dialoguer les divers actants. Il va même jusqu'à souhaiter la création d'un « parlement des choses », dans lequel celles-ci seraient représentées par des personnes qualifiées dans un domaine particulier, tout comme les députés représentent aujourd'hui les citoyens. Donna Haraway montre à travers la métaphore du cyborg ${ }^{40}$ que des choses qui semblent naturelles, comme le corps humain, dépendent en fait de ce que nous en pensons. Cette idée, évidemment intéressante pour le féminisme, l'est aussi en tant que critique de l'opposition entre naturalité et artificialité, le cyborg étant un être mixte. Peter Sloterdijk, quant à lui, propose de passer de la «philosophie », de la vieille Europe, à la «biosophie » ${ }^{41}$, qui considère la vie comme ce qui ne cesse de devenir en s'auto-organisant, grâce aux systèmes immunitaires permettant l'attaque et la défense. Sloterdijk appelle de ses vœux une théorie générale des systèmes immunitaires et des systèmes communs afin de mieux penser divers modes de coexistences et de partages entre humains et non-humains, et d'une façon générale entre natures et cultures. Citons enfin Philippe Descola, que préoccupent les bouleversements systémiques de l'écosystème terrestre (êtres humains inclus). Pour répondre à l'infériorisation des non-humains (objets, animaux et végétaux, agencements divers...) dans le monde de l'Anthropocène, il envisage que puissent être représentés juridiquement par des personnes mandataires, non pas des êtres non-humains en tant que tels, mais « des écosystèmes, c'est-à-dire des rapports d'un certain type entre des êtres localisés dans des espaces plus ou moins vastes, à des milieux de vie donc, quelle que soit leur nature : des bassins-versants, des massifs montagneux, des villes, des littoraux, des quartiers, des zones écologiquement sensibles, des mers, etc. $^{42}$. »

\section{Remarques conclusives}

Ce qui fascine dans le phénomène vivant, ce n'est pas l'être qui vit sa vie mais toute sa machinerie invisible, révélée par la science ; pas l'être qui existe aujourd'hui sur Terre mais l'être

\footnotetext{
${ }^{39}$ Bruno Latour, Enquête sur les modes d'existence. Une anthropologie des Modernes, Paris, La Découverte, 2012.

${ }^{40}$ Donna Haraway, Manifeste cyborg et autres essais. Sciences, fictions, féminismes, Paris, Exils, 2007.

${ }^{41}$ Peter Sloterdijk, Sphères III. Écumes, Paris, Fayard, 2003.

42 Philippe Descola, « Humain, trop humain », conférence inaugurale du colloque Comment penser l'anthropocène ? organisé par Philippe Descola et Catherine Larrère au Collège de France, les 5-6 novembre 2015.
} 
imaginable dans un ailleurs ou un avant hors d'atteinte; pas l'être naturel mais le vivant synthétique, cette réponse créatrice qui ne met pas fin à la question de l'émergence.

Qu'on les appelle «vies partielles », «vivants synthétiques », « machines vivantes ou semivivantes », «cyborg», etc., ces modes d'existence relèvent aussi des autres vies «xénobiologiques » et de leur degré de légitimité en tant que vies. Il y aurait donc lieu de se demander, à propos de chaque être «partiellement vivant », par quels critères il satisfait ou non à ce qu'on pourrait appeler la condition vivante.

Le monde vivant est intrinsèquement inachevé, et inachevable : ce constat d'ordre scientifique a des implications phénoménologiques et, dans le monde judéo-chrétien, des origines théologiques : en créant l'homme "à son image », le Dieu créateur de la Bible a fait de lui son " associé », indéfiniment chargé d'enrichir le monde inachevé en y exerçant sa créativité de manière libre et responsable. Les bio-artistes d'œuvres qui s'auto-engendrent, seraient-ils les représentants les plus emblématiques de cette conception de l'acte créateur qui laisse à son œuvre la liberté d'évoluer ?

En science comme en art, la notion de «vivant artificiel » s'est banalisée et le «continuum d'interactions entre le cerveau humain et ses artefacts technologiques » est en passe d'advenir. Face à ces recompositions unitaires de la matière vivante et de celle que l'on n'ose plus qualifier d'inerte, il est urgent d'enrichir le potentiel relationnel des humains entre eux comme avec les autres existants, y compris les vies artificielles qui deviendraient utiles au plus grand nombre d'humains et de non-humains. De tels efforts iraient dans le sens de la coévolution qui a toujours présidé à «l'évolution créatrice ${ }^{43}$.

\footnotetext{
${ }^{43}$ Henri Bergson, L'évolution créatrice, Paris, Félix Alcan, 1907.
} 